\title{
Human Endogenous Retrovirus: Their Relationship with Hematological Diseases
}

\author{
Sabrina Fischer, Natalia Echeverria, Juan Cristina and Pilar Moreno* \\ Laboratorio de Virologia Molecular, Centro de Investigaciones Nucleares, Universidad de la Republica, Montevideo, Uruguay \\ *Corresponding author: Pilar Moreno, Facultad de Ciencias, Laboratorio de Virologia Molecular, Centro de Investigaciones Nucleares, Universidad de la Republica, \\ Mataojo 2055, 11400 Montevideo, Uruguay, Tel: +598 2252508 00; Fax: +598 2525 0895; E-mail: pmoreno@cin.edu.uy
}

Received date: Aug 05, 2016; Accepted date: Sep 08, 2016; Published date: Sep 15, 2016

Copyright: (c) 2016 Fischer S et al. This is an open-access article distributed under the terms of the Creative Commons Attribution License, which permits unrestricted use, distribution, and reproduction in any medium, provided the original author and source are credited.

\begin{abstract}
Human endogenous retrovirus (HERVs) integrated in the human genome millions of years ago and became a stable part of the inherited genetic material. Most of these HERVs are dysfunctional due to numerous mutations and thus making it impossible to generate a full, infectious retrovirus particle from a single genetic locus. However, many HERVs are still exceptionally well preserved and maintain Open Reading Frames encoding functional viral proteins. The permanence of HERV's genes along evolution suggests that these elements have proven beneficial to human survival. In this regard, the expression of certain HERV proteins is implicated in important physiological functions, such as placental development. Nevertheless, reactivation of HERVs has frequently been observed in a variety of human tumors suggesting their potential to contribute to malignant progression. Considering the role of HERVs in the carcinogenesis process, the purpose of this mini review is to deepen into HERVs expression and its possible implication in hemato-oncologic disease development.
\end{abstract}

Keywords: Retroviruses; Exogenous; Mutations

\section{Introduction}

Human endogenous retroviruses (HERVs) are genetic remnants of ancient retroviral infections of the germ line which occurred along primate evolution. During the infection process, an exogenous retrovirus infected germ-line cells and its viral genome integrated as a provirus into the cell's chromosomal DNA [1-3]. Provided that the integration process and replication of the inserted virus does not prevent fertilization, the fetus will then carry the retroviral element in all its somatic and germ-line cells, thus, the provirus becomes part of the human genome and it is transmitted to the following generations. To date, approximately $8 \%$ of the human genome is composed of such retroviral sequences $[4,5]$.

Intact HERV sequences share the canonical structure of exogenous retroviruses consisting of an internal region of four essential viral genes (gag, pro, pol, and env); flanked by two Long Terminal Repeats (LTRs) elements. The open reading frames (ORFs) encode viral polyproteins which, after post-translational modification, become the critical structural and functional proteins, such as the reverse transcriptase or the transmembrane envelope, while the LTRs specify promoter, enhancer and polyadenylation signals. Many HERVS also express accesory proteins with potentially important cellular functions that may be relevant to disease development. However, most HERVs are dysfunctional due to numerous mutations such as stop codons or frameshifts, inhibiting the translation of functional proteins and thus making it impossible to generate a full, infectious retrovirus particle from a single genetic locus. In addition, internal viral genes have been removed by recombination of the $5^{\prime}$ and $3^{\prime}$ LTRs, creating an important amount of "solo HERV-LTRs" [6-9].

However, many HERVs are still exceptionally well preserved and maintain ORFs encoding functional viral proteins as well. HERV-K family belongs to the latter and its members not only contain sequences which maintain complete, or near-complete ORFs for all viral polyproteins, but they have also been shown to be transcriptionally active [10-12]. The HML-2 group of HERV-Ks is the most recently acquired in the human genome, having integrated 200.000 to 5 million years ago and because of this, they are the only HERVs specific to the human lineage, and therefore the most conserved ones. Approximately 91 full-length copies of HERV-K (HML-2) have been found per haploid genome and $~ 950$ solo-LTRs $[11,13]$.

Many HERVs are transcribed and translated under normal physiological conditions. Nevertheless, reactivation of HERVs has frequently been observed in a variety of human tumors suggesting their potential to contribute to malignant progression.

\section{HERVs in Health and Disease}

The permanence of HERV's genes along evolution suggests that these elements have proven beneficial to human survival. In this regard, the expression of certain HERV proteins is implicated in important physiological functions, such as placental development. The envelope gene product of members of the HERV-W and HERV-FD families, named Syncitin 1 and 2 respectively, are highly fusogenic glycoproteins that are specifically expressed in the placenta mediating the fusion of trophoblast cells $[14,15]$. Endogenous retroviral elements are also involved in physiological functions regulating the transcription of various genes (such as INSL 4, 1,3-GT, endothelin B receptor tissue-specific salivary amylase [16-20]) as well as providing or enhancing protection mechanisms against exogenous virus infections [21-23].

In addition to their physiological roles, HERV s have been proposed as possible cofactors in the etiology of various autoimmune diseases, neurological disorders and cancer [24]. In reference to autoimmune diseases, there are many reports that indicate a possible role of HERVs in the development of multiple sclerosis (MS) [25-27], rheumatoid arthritis (RA) [28,29] and systemic lupus erythematosus (SLE) [30]. A role for HERVs has also been proposed in neurological and 
Page 2 of 5

neuropsychiatric diseases as diverse as Autistic Spectrum Disorder (ASD), Attention Deficit Hyperactivity Disorder (ADHD), and schizophrenia (SCZ). For SCZ, ASD and ADHD indications are accumulating for involvement of the HERV-W and HERV-H families [31-35]. Further studies of HERVs and their pathogenic role both in autoimmune and neurologic diseases are essential to draw proper conclusions and prove a functional link between them. In contrast to the situation with autoimmune diseases and neurological disorders, the picture concerning HERVs expression in malignant diseases is much clearer. Many reports indicate that some HERVs encoding loci are transcriptionally silent in normal cells, becoming active after malignant transformation. In malignant tissues and cell lines, HERVs expression as RNA, protein, and even viral particles are commonly seen [36-38]. The first clear association of HERV-K expression with human disease came from the discovery of HERV-K (HML-2) encoded viral particles observed to bud from germ cell tumors (GCTs) [39,40]. To date, transcripts of HERVs have been detected by many independent investigators in different tumors: breast cancer [41-46], ovarian cancer [47], lymphoma [42], melanoma [48-50], germ line tumors [51,52], hematological neoplasms [53-55], bladder and prostate cancer [56], primary skin tumors and lymphatic metastases [57,58]. Many cancers exhibit a general state of hypomethylation, thus, HERVs activation during tumorogenesis may also be the result of this epigenetic state $[56,59,60]$.

There are different molecular mechanisms by which HERVs may contribute to the promotion of unhealthy processes:

- Given that HERVs sequences are retro transposons, they have the ability to mobilize and be integrated next to cellular genes whose expression could be consequently altered.

- Through HERVs gene activation and overexpression leading to the production of HERVs proteins that could trigger an autoimmune response or may initiate or maintain carcinogenesis by transcriptional activation of various cellular oncogenes [61,62].

- Through the over-production of potential viral oncogenes such as Rec and Np9 proteins. It is known that both proteins have different binding domains to cellular proteins promoting modifications of different cell signaling pathways [63].

- Through the regulatory sequences of LTRs which can promote the regulation of nearby (proto-) oncogenes or growth factors $[18,64,65]$.

These HERVs mechanisms may be triggered by:

- Epigenetic factors such as DNA hypo methylation which can promote a general or more specific (re)activation of HERV sequences $[66,67]$. The epigenetic changes observed in pathological conditions such as SLE or cancer could be translated into an effect on the activation of some of the retro elements present in our genome, which could have a direct or indirect role in the initiation and clinical evolution of certain chronic diseases $[29,64]$.

- Environmental factors (both exogenous and endogenous) which can facilitate HERV's expression. Exogenous factors are associated with chemicals [68]. UV radiation [69-71] and smoking [72]. Viral interactions such as with Epstein-Barr virus (EBV) [73] or Human Immunodeficiency Virus type 1 (HIV-1) [74,75] were also reported. It has been shown that infection of humans with HIV-1 has profound effects upon the resident HERVs by up regulating transcripts and proteins from diverse classes of endogenous retroviruses [76]. Endogenous factors are associated with the expression of estrogen [77], cytokines [78] and also some transcriptional factors [79-81] (Figure 1).

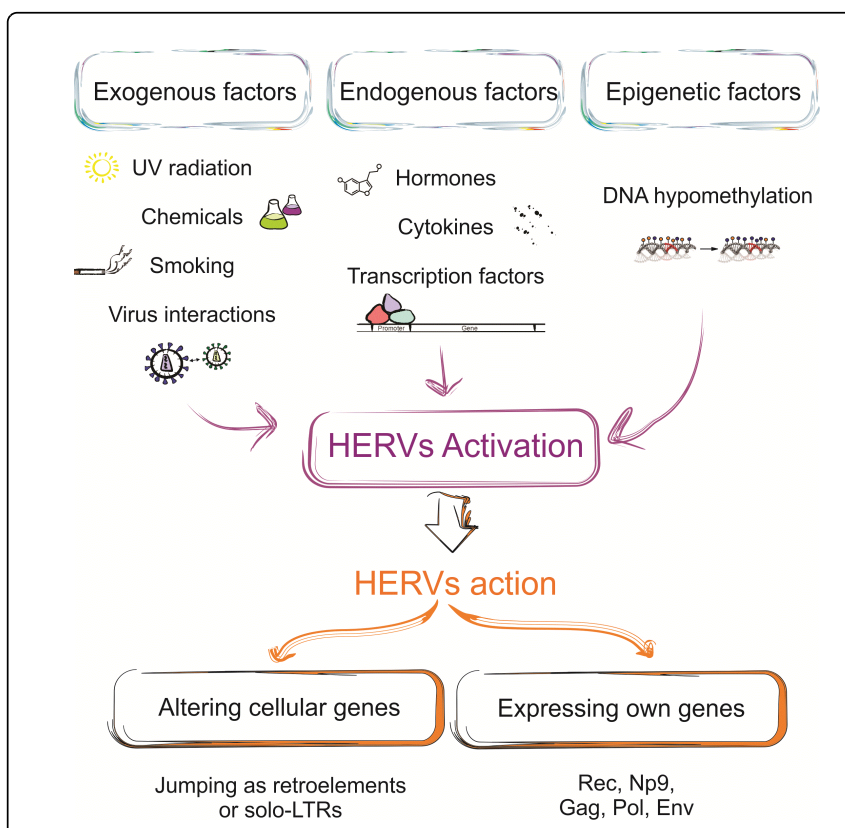

Figure 1: Schematic diagram of the main factors that can trigger HERVs provirus expression, and the main ways through which HERVs can act promoting the development of human diseases. Far from being complete, this is already a quite impressive list.

\section{HERV s and Hemato-oncological Diseases}

With regards to blood-oncological processes, much work has been undertaken to decipher the implications of HERVs expression in the development of different types of blood cancers (Table 1). Many HERVs types as well as many HERVs genes (gag, pol, env, np9) have been associated to a variety of hemato-oncological process and some of them might be related to specific cellular pathways involved in the course of the disease.

The first study that evidenced a relation between the activation of endogenous retroviral sequences and leukemia was performed by McClain and Wilkowski in 1985 [82]. In their work the authors showed for the first time that RNA from HERVs were expressed at higher levels in human lymphoid leukemia cells than in normal lymphocytes. In addition, antibody response against HERV-K peptides has also been reported in leukemia patients suggesting that members of the HERV-K family may be overexpressed in leukemic cells [83].

In the late twentieth century Brodsky and colleagues reported a potential role of HERV-K in both chronic myeloid leukemia (CML) and acute myeloid leukemia (AML) [84]. They found that HERV-K pol gene is expressed in the white blood cells of patients with CML and AML. Consistent with that suggestion, Depil and colleagues found a relative overexpression of HERV-K gag sequences in peripheral blood mononuclear cells (PBMCs) from six out of eight leukemia samples, that included CML, AML, Chronic Lymphoid Leukemia (CLL) and acute lymphoid leukemia (ALL), when compared with healthy PBMCs [53]. Recently it was also reported HERV-K np9 gene overexpression in peripheral blood mononuclear cells (PBMCs) from CLL patients compared to PBMCs from healthy donors, suggesting a significant 
relationship between this pathology and an HERV-K oncoprotein expression [85]. In reference to childhood leukemias, HERVs RNA overexpression was detected in cells from children with ALL as well as HERV-K env gene overexpression in childhood AML [54,86]. In lymphoma patients, extremely high titers of HERV-K viral RNA have been detected in their blood, that fall precipitously when patients are treated. Additionally the presence of HERV-K (HML-2) virus-like particles has been observed [42]. In reference to non-Hodgkin lymphoma (NHL) and in Hodgkin Disease (HD), high levels of HERVK (HML2) RNA in the plasma of patients with HIV positive and HIV negative, but not in normal individuals were detected [87] (Table 1).

\begin{tabular}{|l|l|l|}
\hline Hemato-oncological disease & HERV gene Involved & Reference \\
\hline Chronic myeloid leukemia & HERV-K pol & {$[84]$} \\
\hline Acute myeloid leukemia & $\begin{array}{l}\text { HERV-K pol } \\
\text { HERV-K env }\end{array}$ & {$[86]$} \\
\hline Lymphatic leukemias & HERV-K LTRs & {$[88]$} \\
\hline Chronic lymphocytic leukemia & HERV-K gag & {$[53]$} \\
\hline Chronic myeloid leukemia & HERV-K np9 & {$[90]$} \\
\hline Acute myeloid leukemia & HERman leukemia \\
stem/progenitor cells & HER np9 & {$[85]$} \\
\hline Chronic lymphocytic leukemia & & \\
\hline
\end{tabular}

Table 1: Research studies of HERVs specific mRNA overexpression associated with leukemia.

Taking up the important regulatory role of the solo HERV-K LTRs sequences, their transcripts have been detected in patients with lymphatic leukemias, whereas they have not been found in patients with myelogenous leukemia's or in healthy persons [88].

The HERV-K family is not the only candidate to be involved in the progression of different hemato-oncological diseases. There are other HERVs families, like HERV-F/H, which suggest a cancer-specific expression pattern. An association between HERV-F/H mRNA expression and hematopoietic cancer cell lines, including $\mathrm{B}$ and Myeloid linage leukemia cell lines, has been reported, whereas no expression was observed in normal human tissues [89].

In reference to the pathway altered by HERVs proteins, a recent study identifies Np9 as a potent viral oncogene in human leukemia. Silencing Np9 inhibited the growth of myeloid and lymphoblastic leukemia cells, whereas its expression promoted the growth of leukemia cells in vitro and in vivo. This work proved that Np9 acts as a critical molecular switch of multiple signaling pathways (ERK, AKT, Notch 1 and $\beta$-catenin) promoting the growth of human leukemia stem/progenitor cells [90]. This may be one of the ways which HERVs can contribute to the leukemization process but probably other cellular pathways may be affecting by HERV proteins.

\section{Conclusion}

All this information evidences a relationship between HERVs expression and leukemia. Further studies on the functional roles of HERVs expression in different cellular pathways would be essential to understand the link between HERVs and disease development, and opens new perspective to unravel the etiology of human leukemia. This would not only help us to understand the involvement of these retroviruses in the development of different diseases, but would as well provide useful data on new molecules that could possibly be used as leukemia markers and as potential therapeutic targets.

\section{Acknowledgment}

This work was supported by Agencia Nacional de Investigación e Innovacion (ANII) [project FCE_6569], PEDECIBA, Comision Académica de Posgrados, Universidad de la Republica (UdelaR) and Comisión Sectorial de Investigación Científica (CSIC), UdelaR, Uruguay.

\section{References}

1. Martin MA, Bryan T, Rasheed S, Khan AS (1981) Identification and cloning of endogenous retroviral sequences present in human DNA. Proc Natl Acad Sci USA 78: 4892-4896.

2. Singh SK (2007) Endogenous retroviruses: suspects in the disease world. Future Microbiol 2: 269-275.

3. Feschotte C, Gilbert C (2012) Endogenous viruses: insights into viral evolution and impact on host biology. Nat Rev Genet 13: 283-296.

4. Weiss RA (2006) The discovery of endogenous retroviruses. Retrovirology 3: 67.

5. Balestrieri E, Pica F, Matteucci C, Zenobi R, Sorrentino R, et al. (2015) Transcriptional activity of human endogenous retroviruses in human peripheral blood mononuclear cells. Biomed Res Int.

6. Griffiths DJ (2001) Endogenous retroviruses in the human genome sequence. Genome Biol 2: 1017.

7. Bannert N, Kurth R (2004) Retroelements and the human genome: new perspectives on an old relation. Proc Natl Acad Sci USA 101: 14572-14579.

8. Downey RF, Sullivan FJ, Wang-Johanning F, Ambs S, Giles FJ, et al. (2015) Human endogenous retrovirus $\mathrm{K}$ and cancer: Innocent bystander or tumorigenic accomplice? Int J Cancer 137: 1249-1257.

9. Hughes JF, Coffin JM (2004) Human endogenous retrovirus K solo-LTR formation and insertional polymorphisms: implications for human and viral evolution. Proc Natl Acad Sci USA 101: 1668-1672.

10. Bannert N, Kurth R (2006) The evolutionary dynamics of human endogenous retroviral families. Annu Rev Genomics Hum Genet 7: 149-173.

11. Subramanian RP, Wildschutte JH, Russo C, Coffin JM (2011) Identification, characterization, and comparative genomic distribution of the HERV-K (HML-2) group of human endogenous retroviruses. Retrovirology 8: 90.

12. Berkhout B, Jebbink M, Zsiros J (1999) Identification of an Active Reverse Transcriptase Enzyme Encoded by a Human Endogenous HERVK Retrovirus. J Virol 73: 2365-2375.

13. Barbulescu M, Turner G, Seaman MI, Deinard AS, Kidd KK, et al. (1999) Many human endogenous retrovirus K (HERV-K) proviruses are unique to humans. Curr Biol 9: 861-868.

14. Blaise S, Ruggieri A, Dewannieux M, Cosset FL, Heidmann T (2004) Identification of an envelope protein from the FRD family of human endogenous retroviruses (HERV-FRD) conferring infectivity and functional conservation among simians. J Virol 78: 1050-1054.

15. Mi S, Lee X, Li XP, Veldman GM, Finnerty H, et al. (2000) Syncytin is a captive retroviral envelope protein involved in human placental morphogenesis. Nature 403: 785-789.

16. Bieche I, Laurent A, Laurendeau I, Duret L, Giovangrandi Y, et al. (2003) Placenta-specific INSL4 expression is mediated by a human endogenous retrovirus element. Biol Reprod 68: 1422-1429.

17. Jern P, Coffin JM (2008) Effects of retroviruses on host genome function. Annu Rev Genet 42: 709-732. 
18. Dunn CA, Medstrand P, Mager DL (2003) An endogenous retroviral long terminal repeat is the dominant promoter for human beta1,3galactosyltransferase 5 in the colon. Proc Natl Acad Sci USA 100: $12841-12846$.

19. Medstrand P, Landry JR, Mager DL (2001) Long terminal repeats are used as alternative promoters for the endothelin $\mathrm{B}$ receptor and apolipoprotein C-I genes in humans. J Biol Chem 276: 1896-1903.

20. Ting CN, Rosenberg MP, Snow CM, Samuelson LC, Meisler MH, et al. (1992) Endogenous retroviral sequences are required for tissue-specific expression of a human salivary amylase gene. Genes Dev 6: 1457-1465.

21. Garrison KE, Jones RB, Meiklejohn DA, Anwar N, Ndhlovu LC, et al. (2007) $\mathrm{T}$ cell responses to human endogenous retroviruses in HIV-1 infection. PLoS Pathog 3: 1617-1627.

22. Gonzalez-Hernandez MJ, Swanson MD, Galindo CR, Cookinham S, King SR, et al. (2012) Expression of Human Endogenous Retrovirus Type-K (HML-2) is activated by the Tat protein of HIV-1. J Virol 86: 7790-7805.

23. Chuong EB, Elde NC, Feschotte C (2016) Regulatory evolution of innate immunity through co-option of endogenous retroviruses. Science 351: 1083-1087.

24. Moyes D, Griffiths DJ, Venables PJ (2007) Insertional polymorphisms: a new lease of life for endogenous retroviruses in human disease. Trends Genet 23: 326-333.

25. Christensen $\mathrm{T}$ (2005) Association of human endogenous retroviruses with multiple sclerosis and possible interactions with herpes viruses. Rev Med Virol 15: 179-211.

26. Rasmussen HB, Geny C, Deforges L, Perron H, Tourtelotte W, et al. (1995) Expression of endogenous retroviruses in blood mononuclear cells and brain tissue from multiple sclerosis patients. Mult Scler 1: 82-87.

27. Garcia-Montojo M, Dominguez-Mozo M, Arias-Leal A, Garcia-Martinez A, de las Heras V, et al. (2013) The DNA Copy Number of Human Endogenous Retrovirus-W (MSRV-Type) Is Increased in Multiple Sclerosis Patients and Is Influenced by Gender and Disease Severity. PLoS One.

28. Nakagawa K, Brusic V, McColl G, Harrison L (1997) Direct evidence for the expression of multiple ERVs in the synovial compartment in rheumatoid arthritis. Arthritis Rheum 40: 627-638.

29. Balada E, Ordi-Ros J, Vilardell-Tarres M (2009) Molecular mechanisms mediated by Human Endogenous Retroviruses (HERVs) in autoimmunity. Rev Med Virol 19: 273-286.

30. Blomberg J, Nived O, Pipkorn R, Bengtsson A, Erlinge D, et al. (1994) Increased antiretroviral antibody reactivity in sera from a defined population of patients with systemic lupus erythematosus: Correlation with autoantibodies and clinical manifestations. Arthritis Rheum 37: 57-66.

31. Christensen $\mathrm{T}$ (2016) Human endogenous retroviruses in neurologic disease. Apmis 124: 116-126.

32. Balestrieri E, Arpino C, Matteucci C, Sorrentino R, Pica F, et al. (2012) HERVs Expression in Autism Spectrum Disorders.

33. Balestrieri E, Pitzianti M, Matteucci C, DAgati E, Sorrentino R, et al. (2013) Human endogenous retroviruses and ADHD. World J Biol Psychiatry 1-6.

34. Karlsson H, Bachmann S, Schroder J, McArthur J, Torrey EF, et al. (2001) Retroviral RNA identified in the cerebrospinal fluids and brains of individuals with schizophrenia. Proc Natl Acad Sci 98: 4634-4639.

35. Slokar G, Hasler G (2016) Human endogenous retroviruses as pathogenic factors in the development of schizophrenia. Front Psychiatry.

36. Kassiotis G (2014) Endogenous retroviruses and the development of cancer. J Immunol 192: 1343-1349.

37. Bhardwaj N, Coffin JM (2014) Endogenous retroviruses and human cancer: Is there anything to the rumors? Cell Host Microbe 15: 255-259.

38. Lower R (1999) The pathogenic potential of endogenous retroviruses: Facts and fantasies. Trends Microbiol 7: 350-356.

39. Ruprecht K, Mayer J, Sauter M, Roemer K, Mueller-Lantzsch N (2008) Endogenous retroviruses. Cell Mol Life Sci 65: 3366-3382.
40. Flockerzi A, Ruggieri A, Frank O, Sauter M, Maldener E, et al. (2008) Expression patterns of transcribed human endogenous retrovirus HERVK(HML-2) loci in human tissues and the need for a HERV Transcriptome Project. BMC Genomics 9: 354.

41. Ejthadi HD, Martin JH, Junying J, Roden DA, Lahiri M, et al. (2005) A novel multiplex RT-PCR system detects human endogenous retrovirus-K in breast cancer. Arch Virol 150: 177-184.

42. Contreras-Galindo R, Kaplan MH, Leissner P, Verjat T, Ferlenghi I, et al. (2008) Human endogenous retrovirus K (HML-2) elements in the plasma of people with lymphoma and breast cancer. J Virol 82: 9329-9336.

43. Wang-Johanning F, Frost AR, Johanning GL, Khazaeli MB, LoBuglio AF, et al. (2001) Expression of human endogenous retrovirus $k$ envelope transcripts in human breast cancer. Clin Cancer Res 7: 1553-1560.

44. Wang-Johanning F, Frost AR, Jian B, Epp L, Lu DW, et al. (2003) Quantitation of HERV-K env gene expression and splicing in human breast cancer. Oncogene 22: 1528-1535.

45. Wang-Johanning F, Radvanyi L, Rycaj K, Plummer JB, Yan P, et al. (2008) Human endogenous retrovirus $\mathrm{K}$ triggers an antigen-specific immune response in breast cancer patients. Cancer Res 68: 5869-5877.

46. Wang-Johanning F, Rycaj K, Plummer JB, Li M, Yin B, et al. (2012) Immunotherapeutic potential of anti-human endogenous retrovirus- $k$ envelope protein antibodies in targeting breast tumors. J Natl Cancer Inst 104: $189-210$

47. Wang-Johanning F, Liu J, Rycaj K, Huang M, Tsai K, et al. (2007) Expression of multiple human endogenous retrovirus surface envelope proteins in ovarian cancer. Int J Cancer 120: 81-90.

48. Schiavetti F, Thonnard J, Colau D, Boon T, Coulie PG (2002) A human endogenous retroviral sequence encoding an antigen recognized on melanoma by cytolytic T lymphocytes. Cancer Res 62: 5510-5516.

49. Serafino A, Balestrieri E, Pierimarchi P, Matteucci C, Moroni G, et al. (2009) The activation of human endogenous retrovirus K (HERV-K) is implicated in melanoma cell malignant transformation. Exp Cell Res 315: 849-862.

50. Singh S, Kaye S, Gore ME, McClure MO, Bunker CB, et al. (2009) The role of human endogenous retroviruses in melanoma. Br J Dermatol 161: 1225-1231.

51. Kleiman A, Senyuta N, Tryakin A, Sauter M, Karseladze A, et al. (2004) HERV-K(HML-2) GAG/ENV antibodies as indicator for therapy effect in patients with germ cell tumors. Int J Cancer 110: 459-461.

52. Herbst H, Sauter M, Mueller-Lantzsch N (1996) Expression of human endogenous retrovirus $\mathrm{K}$ elements in germ cell and trophoblastic tumors. Am J Pathol 149: 1727-1735.

53. Depil S, Roche C, Dussart P, Prin L (2002) Expression of a human endogenous retrovirus, HERV-K, in the blood cells of leukemia patients. Leukemia 16: 254-259.

54. Iwabuchi H, Kakihara T, Kobayashi T, Imai C, Tanaka A, et al. (2004) A gene homologous to human endogenous retrovirus overexpressed in childhood acute lymphoblastic leukemia. Leuk Lymphoma 45: 2303-2306.

55. Boyd MT, Foley B, Brodsky I (1997) Evidence for copurification of HERV-K-related transcripts and a reverse transcriptase activity in human platelets from patients with essential thrombocythemia. Blood 90: 40224030.

56. Goering W, Ribarska T, Schulz WA (2011) Selective changes of retroelement expression in human prostate cancer. Carcinogenesis 32: 1484-1492.

57. Muster T, Waltenberger A, Grassauer A, Hirschl S, Caucig P, et al. (2003) An Endogenous Retrovirus Derived from Human Melanoma Cells. Cancer Res 63: 8735-8741.

58. Buscher K, Trefzer U, Hofmann M, Sterry W, Kurth R, et al. (2005) Expression of human endogenous retrovirus $\mathrm{K}$ in melanomas and melanoma cell lines. Cancer Res 65: 4172-4180.

59. Stengel S, Fiebig U, Kurth R, Denner J (2010) Regulation of human endogenous retrovirus- $\mathrm{K}$ expression in melanomas by $\mathrm{CpG}$ methylation. Genes Chromosom Cancer 49: 401-411. 
60. Iramaneerat $\mathrm{K}$, Rattanatunyong $\mathrm{P}$, Khemapech $\mathrm{N}$, Triratanachat $\mathrm{S}$, Mutirangura A (2011) HERV-K hypomethylation in ovarian clear cell carcinoma is associated with a poor prognosis and platinum resistance. Int J Gynecol Cancer 21: 51-57.

61. Tai AK, O’Reilly EJ, Alroy KA, Simon KC, Munger KL, et al. (2008) Human endogenous retrovirus-K18 Env as a risk factor in multiple sclerosis. Mult Scler 14: 1175-1180.

62. Romanish MT, Cohen CJ, Mager DL (2010) Potential mechanisms of endogenous retroviral-mediated genomic instability in human cancer. Semin Cancer Biol 20: 246-253.

63. Denne M, Sauter M, Armbruester V, Licht JD, Roemer K, et al. (2007) Physical and functional interactions of human endogenous retrovirus proteins $\mathrm{Np} 9$ and $\mathrm{rec}$ with the promyelocytic leukemia zinc finger protein. J Virol 81: 5607-5616.

64. Mullins CS, Linnebacher M (2012) Human endogenous retroviruses and cancer: Causality and therapeutic possibilities. World J Gastroenterol 18: 6027-6035.

65. Lamprecht B, Walter K, Kreher S, Kumar R, Hummel M, et al. (2010) Derepression of an endogenous long terminal repeat activates the CSF1R proto-oncogene in human lymphoma. Nat Med 16: 571-579.

66. Schulz WA, Steinhoff C, Florl AR (2006) Methylation of endogenous human retroelements in health and disease. Curr Top Microbiol Immunol 310: 211-250.

67. Jones PA, Baylin SB (2002) The fundamental role of epigenetic events in cancer. Nat Rev Genet 3: 415-428

68. Khan AS, Muller J, Sears JF (2001) Early detection of endogenous retroviruses in chemically induced mouse cells. Virus Res 79: 39-45.

69. Hohenadl C, Germaier H, Walchner M, Hagenhofer M, Herrmann M, et al. (1999) Transcriptional activation of endogenous retroviral sequences in human epidermal keratinocytes by UVB irradiation. J Invest Dermatol 113: 587-594.

70. Schanab O, Humer J, Gleiss A, Mikula M, Sturlan S, et al. (2011) Expression of human endogenous retrovirus $\mathrm{K}$ is stimulated by ultraviolet radiation in melanoma. Pigment Cell Melanoma Res 24: 656-665.

71. Reiche J, Pauli G, Ellerbrok H (2010) Differential expression of human endogenous retrovirus $\mathrm{K}$ transcripts in primary human melanocytes and melanoma cell lines after UV irradiation. Melanoma Res 20: 435-440.

72. Gabriel U, Steidler A, Trojan L, Michel MS, Seifarth W, et al. (2010) Smoking increases transcription of human endogenous retroviruses in a newly established in vitro cell model and in normal urothelium. AIDS Res Hum Retroviruses 26: 883-888.

73. Sutkowski N, Chen G, Calderon G, Huber BT (2004) Epstein-Barr virus latent membrane protein LMP-2A is sufficient for transactivation of the human endogenous retrovirus HERV-K18 superantigen. J Virol 78: 7852-7860.

74. Contreras-Galindo R, Lopez P, Velez R, Yamamura Y (2007) HIV-1 infection increases the expression of human endogenous retroviruses type $\mathrm{K}$ (HERV-K) in vitro. AIDS Res Hum Retroviruses 23: 116-122.

75. Contreras-Galindo R, Kaplan MH, Contreras-Galindo AC, GonzalezHernandez MJ, Ferlenghi I, et al. (2012) Characterization of Human Endogenous Retroviral Elements in the Blood of HIV-1-Infected Individuals. J Virol 86: 262-276.
76. Van der Kuyl AC (2012) HIV infection and HERV expression: a review. Retrovirology 9: 6 .

77. Ono M, Kawakami M, Ushikubo H (1987) Stimulation of expression of the human endogenous retrovirus genome by female steroid hormones in human breast cancer cell line T47D. J Virol 61: 2059-2062.

78. Katsumata K, Ikeda H, Sato M, Ishizu A, Kawarada Y, et al. (1999) Cytokine regulation of env gene expression of human endogenous retrovirus- $\mathrm{R}$ in human vascular endothelial cells. Clin Immunol Orlando Fla 93: 75-80.

79. Katoh I, Mirova A, Kurata S, Murakami Y, Horikawa K, et al. (2011) Activation of the long terminal repeat of human endogenous retrovirus $\mathrm{K}$ by melanoma-specific transcription factor MITF-M. Neoplasia 13: 1081-1092.

80. Fuchs NV, Kraft M, Tondera C, Hanschmann KM, Lower J, et al. (2011) Expression of the Human Endogenous Retrovirus (HERV) Group HML-2/HERV-K Does Not Depend on Canonical Promoter Elements but Is Regulated by Transcription Factors Sp1 and Sp3. J Virol 85: 3436-3448.

81. Knossl M, Lower R, Löwer J (1999) Expression of the human endogenous retrovirus HTDV/HERV-K is enhanced by cellular transcription factor YY1. J Virol 73: 1254-1261.

82. McClain K, Wilkowski C (1985) Activation of endogenous retroviral sequences in human leukemia. Biochem Biophys Res Commun 133: 945-950.

83. Denner J, Phelps R, Lower R, Lower J, Kurth R, et al. (1995) Expression of the human endogenous retrovirus HERV-K in tumor and normal tissues and antibody response of pregnant women, tumor and AIDS patients against HERV-K Gag and Env peptides. AIDS Res Hum Retroviruses 11: 103-107.

84. Brodsky I, Foley B, Gillespie D (1993) Expression of human endogenous retrovirus (HERV-K) in chronic myeloid leukemia. Leuk Lymphoma 11 Suppl 1: 119-123.

85. Fischer S, Echeverria N, Moratorio G, Landoni AI, Dighiero G, et al. (2014) Human endogenous retrovirus np9 gene is over expressed in chronic lymphocytic leukemia patients. Leuk Res reports 3: 70-72.

86. Januszkiewicz-Lewandowska D, Nowicka K, Rembowska J, Fichna M, Zurawek M, et al. (2013) Env gene expression of human endogenous retrovirus- $\mathrm{k}$ and human endogenous retrovirus- $\mathrm{W}$ in childhood acute leukemia cells. Acta Haematol 129: 232-237.

87. Gitlin SD, Contreras-Galindo R, Kaplan MH, Markovitz DM (2008) Role of Human Endogenous Retroviruses in Lymphoma Pathogenesis and a Possible Biomarker of Disease. Blood 112: 3751.

88. Simon M, Haltmeier M, Papakonstantinou G, Werner T, Hehlmann R, et al. (1994) Transcription of HERV-K-related LTRs in human placenta and leukemic cells. Leukemia 8 Suppl 1: S12-S17.

89. Patzke S, Lindeskog M, Munthe E, Aasheim HC (2002) Characterization of a Novel Human Endogenous Retrovirus, HERV-H/F, Expressed in Human Leukemia Cell Lines. Virology 303: 164-173.

90. Chen T, Meng Z, Gan Y, Wang X, Xu F, et al. (2013) The viral oncogene $\mathrm{Np} 9$ acts as a critical molecular switch for co-activating $\beta$-catenin, ERK, Akt and Notch1 and promoting the growth of human leukemia stem/ progenitor cells. Leuk Off J Leuk Soc Am Leuk Res Fund 27: 1469-1478. 